\title{
4-芳亚甲基-2-羟基-3-蒎酮类化合物的合成及其紫外吸收特征研究
}

\author{
魏柏松 ${ }^{a}$ 徐 徐 ${ }^{a, b}$ 杨益琴 ${ }^{c} \quad$ 曹晓琴 ${ }^{a}$ 王石发 ${ }^{*, a, b}$ \\ ( ${ }^{a}$ 南京林业大学化学工程学院 南京 210037) \\ ( ${ }^{b}$ 江苏省生物质绿色燃料与化学品重点实验室 南京 210037) \\ ( ${ }^{c}$ 南京林业大学轻工科学与工程学院 南京 210037)
}

\begin{abstract}
摘要 以 $(-)-\alpha$-蒎烯为原料合成了系列新型 4-芳亚甲基-2-羊基-3-蒎酮类化合物. (-)- $\alpha$-蒎烯经选择性氧化得到 $(+)-2-$ 羟基-3-蒎酮; 在碱催化作用下, $(+)-2$-羟基-3-蒎酮与苯甲醛、对甲基苯甲醛、对甲氧基苯甲醛、对羟基苯甲醛、对氯苯

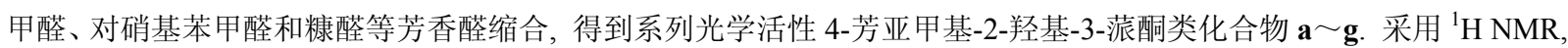
${ }^{13} \mathrm{C}$ NMR, GC-MS 和 FT-IR 等分析手段对合成所得 4-芳亚甲基-2-羊基-3-蒎酮类化合物的结构进行了表征, 考察了它们 的紫外吸收特性及光稳定性. 结果表明, 化合物 $\mathbf{a}, \mathbf{b}, \mathbf{e}$ 对 UVB 具有良好的吸收性能; 而化合物 $\mathbf{c}, \mathbf{d}, \mathbf{f}, \mathbf{g}$ 兼具长波紫外 线(UVA)和中波紫外线(UVB)的吸收性能. 化合物 $\mathbf{a} \sim \mathbf{g}$ 的光稳定性顺序为 $\mathbf{d}>\mathbf{c}>\mathbf{e}>\mathbf{f}>\mathbf{b}>\mathbf{a}>\mathbf{g}$.
\end{abstract} 关键词 (-)- $\alpha$-蒎烯; (+)-2-羟基-3-蒎酮; 4-芳亚甲基-2-差基-3-蒎酮; 紫外吸收

\section{Synthesis and Ultraviolet Absorption Characteristics of 4-Arylidene-2-hydroxy-3-pinanones}

\author{
Wei, Baisong $^{a} \quad \mathrm{Xu}, \mathrm{Xu}^{a, b} \quad$ Yang, Yiqin $^{\mathrm{c}} \quad \mathrm{Cao}, \mathrm{Xiaoqin}^{a} \quad$ Wang, Shifa ${ }^{*, a, b}$ \\ ( ${ }^{a}$ College of Chemical Engineering, Nanjing Forestry University, Nanjing 210037) \\ ( ${ }^{b}$ Jiangsu Key Laboratory of Biomass-based Green Fuels and Chemicals, Nanjing 210037) \\ ( ${ }^{c}$ College of Light Industry and Engineering, Nanjing Forestry University, Nanjing 210037)
}

\begin{abstract}
A new series of 4-arylidene-2-hydroxy-3-pinanones were synthesized from ( -$)$ - $\alpha$-pinene. (+)-2-Hydroxy3 -pinanone was obtained from $(-)-\alpha$-pinene by selective oxidation with potassium permanganate, and it was reacted with aromatic aldehydes including benzaldehyde, $p$-methylbenzaldehyde, $p$-methoxybenzaldehyde, $p$-hydroxybenzaldehyde, $p$-chlorobenzaldehyde, $p$-nitrobenzaldehyde, and furfural catalyzed with alkali catalysts to get optical activity 4-arylidene-2-hydroxy-3-pinanones $\mathbf{a} \sim \mathbf{g}$. The structures of $\mathbf{a} \sim \mathbf{g}$ were identified by means of ${ }^{1} \mathrm{H}$ NMR, ${ }^{13} \mathrm{C}$ NMR, GC-MS and FT-IR techniques, and their ultraviolet absorption characteristics and light stabilities were also investigated. The results showed that compounds a, b and e exhibit good absorbency as the UV-B-type absorbents, and compounds $\mathbf{c}, \mathbf{d}, \mathbf{f}$ and $\mathbf{g}$ had both functions as long-wave ultraviolet (UVA) and medium-wave ultraviolet (UVB) absorbents. The light stability sequence of these compounds was $\mathbf{d}>\mathbf{c}>\mathbf{e}>\mathbf{f}>\mathbf{b}>\mathbf{a}>\mathbf{g}$.
\end{abstract}

Keywords (-)- $\alpha$-pinene; (+)-2-hydroxy-3-pinanone; 4-arylidene-2-hydroxy-3-pinanones; ultraviolet absorption

近年来随着大气臭氧层的不断破坏，增加的紫外线 辐射量已成为当今最关注的全球性环境问题之一，对人 类健康产生了严重的影响 ${ }^{[1,2]}$. 320 400 nm 的长波紫外 线(UVA)，其能量可及皮肤真皮层，给血管壁或结合组 织中的弹性纤维带来缓慢的变化, 从而引起皮肤的褐色 化，皮肤弹性下降，促进皱纹的发生，使人急剧老化 ${ }^{[3,4]}$, 另外还能促进红斑反应甚至引发光毒性或光敏反应 ${ }^{[5]}$.
280 320 nm 的中波长紫外线(UVB)能引起皮肤产生红 斑或水疮，促进黑色素形成，使皮肤产生色素沉着，致 使褐斑的形成, 是主要的防范对象 ${ }^{[6,7]}$. 对于敏感性皮肤 在日光下连续经过 UVA 和 UVB 的辐射还能损伤 DNA, 使免疫力下降，甚至诱发皮肤癌. 所以防御过量的UVA 和 UVB 照射，保护人体免受伤害，延缓衰老十分重 要 ${ }^{[8,9]}$.

\footnotetext{
*E-mail: wangshifa65@yahoo.com.cn

Received July 26, 2012; revised August 23, 2012; published online September 4, 2012

Project supported by the National Natural Science Foundation of China (No. 31170538).

国家自然科学基金(No. 31170538)资助项目.
} 
目前可用作化妆品紫外线吸收剂的萜烯类化合物 主要是樟脑衍生物, 包括 3-亚芐基樟脑、4-甲基苄亚基 樟脑、亚苠基樟脑磺酸、对苯二亚甲基二樟脑磺酸等衍 生物 ${ }^{[10 ~ 14]}$. 合成樟脑作为萜烯类化合物, 其生产过程存 在合成路线长、环境污染大等缺点, 而天然樟脑则存在 价格高、来源少等不足之处 ${ }^{[15]}$. 为此, 开发新型的萜烯 类紫外线吸收剂尤为紧迫.

本文以廉价易得的生物质资源 $\alpha$-蒎烯为原料 ${ }^{[16,17]}$, 经选择性氧化制得具有与樟脑相似的六元双环结构的 2-差基-3-蒎酮, 再与不同结构的芳香醛进行羟醀缩合反 应, 合成系列衍生物, 并探索化合物结构与紫外吸收效 果之间的关系, 以求篮选出紫外吸收效果好、紫外吸收 光谱范围广、稳定性好、合成操作简便经济、得率高、 污染少的新型紫外线吸收剂, 对拓宽我国松节油的利用 具有重要意义. 所合成的化合物通过 ${ }^{1} \mathrm{H} N \mathrm{NMR},{ }^{13} \mathrm{C}$ NMR, GC-MS 和 FT-IR 表征确证其结构, 合成线路见 Scheme 1.

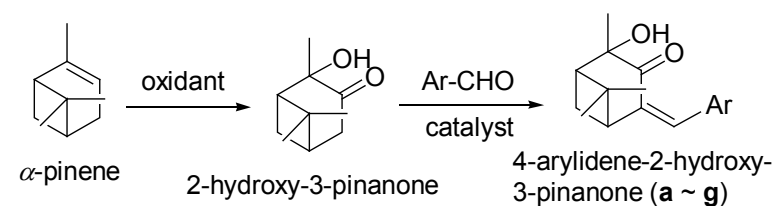

$\mathrm{Ar}=\mathrm{Ph}(\mathbf{a}), p-\mathrm{CH}_{3} \mathrm{C}_{6} \mathrm{H}_{4}(\mathbf{b}), p-\mathrm{CH}_{3} \mathrm{OC}_{6} \mathrm{H}_{4}(\mathbf{c}), p-\mathrm{HOC}_{6} \mathrm{H}_{4}(\mathbf{d})$, $p-\mathrm{ClC}_{6} \mathrm{H}_{4}(\mathbf{e}), p-\mathrm{O}_{2} \mathrm{NC}_{6} \mathrm{H}_{4}(\mathbf{f})$, 2-furyl (g)
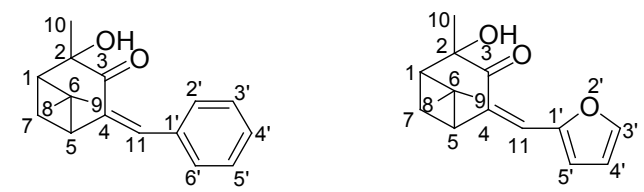

Scheme 1

\section{1 实验部分}

\section{1 仪器与试剂}

德国 Bruker AV $500 \mathrm{MHz}$ 核磁共振仪(TMS 为内标, $\mathrm{CDCl}_{3}$ 或 DMSO 为溶剂); 美国 Agilent 7890A 气相色谱 仪; 美国 Agilent 5975C 质谱仪; Nicolet 380 FT-IR 红外 光谱仪; 日本 Shimadzu UV-2450 紫外分光光度计; 北京 泰克 X-6 显微熔点测定仪(温度计未经校正); 上海精密 科学 $\mathrm{WZZ}-2 \mathrm{~S}$ 数字式自动旋光仪.

$(-)-\alpha$-蒎烯购自江西省吉水县宏达天然香料有限 公司, 经减压精密分馏(回流比为 $20: 1, V: V$ ), 收集 $46 \sim 49{ }^{\circ} \mathrm{C} / 32.0 \mathrm{kPa}$ 的馏分作为合成所用原料, $\mathrm{GC}$ 纯度 达 $98.0 \%$ ( $[\alpha]_{\mathrm{D}}^{20}-47.0$, neat); 苯甲醛、对甲基苯甲醛、 对甲氧基苯甲醛、对羟基苯甲醛、对氯苯甲醛、对硝基 苯甲醛、糠醛均购自国药集团化学试剂有限公司, 分析 纯; 其他试剂和溶剂均为市售分析纯, 未处理直接使用.

\subsection{2-羟基-3-蒎酮的合成}

在配有摚拌器、温度计和加料漏斗的 $500 \mathrm{~mL}$ 三口 烧瓶中, 依次加入 $(-)-\alpha$-蒎烯 $13.60 \mathrm{~g}(0.1 \mathrm{~mol})$, 丙酮 $110 \mathrm{~mL}$ 和去离子水 $12 \mathrm{~mL}$, 冰浴冷却至 $0 \sim 5{ }^{\circ} \mathrm{C}$ 左右, 分批加入 $31.61 \mathrm{~g}(0.2 \mathrm{~mol})$ 充分碾细的高锰酸钾 $(1.5 \sim 2$ $\mathrm{h}$ 内加完, 控制反应体系温度, 防止温度过高冲料). 在 室温下继续反应 $6 \mathrm{~h}$ (GC 跟踪监测). 反应结束后, 用砂 芯漏斗过滤，再用丙酮 $(10 \mathrm{~mL} \times 3)$ 洗涤固体残渣. 用旋 转浓缩仪回收丙酮, 剩余物中加入 $30 \mathrm{~mL}$ 乙酸乙酯, 用 饱和食盐水洗涤至中性, 经无水硫酸钠干燥、过滤、浓 缩后, 再减压蒸馏, 收集 $120 \sim 122{ }^{\circ} \mathrm{C} / 2.66 \mathrm{kPa}$ 馏分, 得 到 $12.72 \mathrm{~g}$ 无色固体, 产率 $75.6 \%$, 纯度为 $98.3 \%(\mathrm{GC})$. m.p. $38.1 \sim 39.0{ }^{\circ} \mathrm{C}$; $[\alpha]_{\mathrm{D}}^{25}+37.5\left(\right.$ c $\left.0.5, \mathrm{CHCl}_{3}\right) ;{ }^{1} \mathrm{H}$ NMR $\left(\mathrm{CDCl}_{3}, 500 \mathrm{MHz}\right) \delta: 0.74$ (s, 3H, 9-CH $\left.)_{3}\right), 1.22$ (s, $\left.3 \mathrm{H}, 8-\mathrm{CH}_{3}\right), 1.23\left(\mathrm{~s}, 3 \mathrm{H}, 10-\mathrm{CH}_{3}\right), 1.58(\mathrm{~d}, J=11.0 \mathrm{~Hz}, 1 \mathrm{H}$, $7 \alpha-\mathrm{CH}), 1.94 \sim 1.98$ (m, $2 \mathrm{H}, 7 \beta-\mathrm{CH}, 4 \alpha-\mathrm{CH}), 2.27 \sim 2.29$ $(\mathrm{m}, 1 \mathrm{H}, 5-\mathrm{CH}), 2.46(\mathrm{~s}, 2 \mathrm{H}, 1-\mathrm{CH}, 4 \beta-\mathrm{CH}), 3.02(\mathrm{~s}, 1 \mathrm{H}$, $\mathrm{OH}) ;{ }^{13} \mathrm{C} \mathrm{NMR}\left(\mathrm{CDCl}_{3}, 500 \mathrm{MHz}\right) \delta: 22.5,24.8,27.0$, 28.1, 38.1, 38.9, 42.7, 49.6, 76.6, 213.6; FT-IR (KBr) $v$ : $3449\left(v_{\mathrm{O}-\mathrm{H}}\right), 2975\left(v_{\text {as C}-\mathrm{H}}, \mathrm{CH}_{3}\right), 2917\left(v_{\text {as C}-\mathrm{H}}, \mathrm{CH}_{2}\right), 2872$ $\left(v_{\mathrm{s} C-\mathrm{H}}, \mathrm{CH}_{3}\right), 1716\left(v_{\mathrm{C}=\mathrm{O}}\right), 1472\left(\delta_{\mathrm{sC}-\mathrm{H}}, \mathrm{CH}_{2}\right), 1370\left(\delta_{\mathrm{sC}-\mathrm{H}}\right.$, $\left.\mathrm{CH}_{3}\right), 1326\left(\delta_{\mathrm{C}-0}\right), 1165\left(v_{\text {as }} \mathrm{C}^{-0}, \mathrm{C}-\mathrm{OH}\right) \mathrm{cm}^{-1}$; EI-MS (70 eV) $m / z(\%): 168\left(\mathrm{M}^{+}, 3\right), 153\left(\mathrm{M}^{+}-\mathrm{CH}_{3}, 1\right), 150$ $\left(\mathrm{M}^{+}-\mathrm{H}_{2} \mathrm{O}, 1\right), 140\left(\mathrm{M}^{+}-\mathrm{CO}, 3\right), 125\left(\mathrm{M}^{+}-\mathrm{CO}-\mathrm{CH}_{3}\right.$, 14), 111 (19), 99 (100), 83 (11), 81 (14), 71 (99), 55 (15).

\subsection{4-芳亚甲基-2-羟基-3-蒎酮衍生物 $\mathrm{a} \sim \mathrm{g}$ 的合成}

在配有氮气保护装置、温度计和冷凝器的 $50 \mathrm{~mL}$ 三 口烧瓶中, 依次加入 $1.68 \mathrm{~g}(10.0 \mathrm{mmol}) 2$-差基-3-蒎酮, $1.27 \mathrm{~g}(12.0 \mathrm{mmol})$ 苯甲醛, $10 \mathrm{~mL}$ 叔丁醇, 在电磁搅拌下 加入 $0.30 \mathrm{~g}$ 叔丁醇钾(醛酮总质量的 $10 \%$ )后, 升温至 70 ${ }^{\circ} \mathrm{C}$ 反应 $4 \mathrm{~h}, 2$-差基-3-蒎酮转化率达 $97.9 \%$, 反应选择性 达 $97.5 \%$ ( $\mathrm{GC}$ 跟踪监测). 冷却后, 反应液中加入 $30 \mathrm{~mL}$ 饱和食盐水, 用 $60 \mathrm{~mL}$ 乙酸乙酯分 3 次萃取, 合并的有 机相用饱和食盐水洗至中性, 经无水硫酸钠干燥、过滤、 浓缩后得到红棕色粘稠物. 粗产物经 $10 \mathrm{~mL}$ 乙酸乙酯/ $20 \mathrm{~mL}$ 石油醚重结晶得 $1.88 \mathrm{~g}$ 无色透明晶体 4-苯亚甲 基-2-羑基-3-蒎酮(a), 产率 73.4\%, 纯度为 $99.5 \%(\mathrm{GC})$. m.p. $103.5 \sim 104.5{ }^{\circ} \mathrm{C}$; $[\alpha]_{\mathrm{D}}^{20}+18.4\left(c 0.5, \mathrm{CH}_{3} \mathrm{OH}\right)$.

4-(4'-甲基苯亚甲基)-2-羟基-3-蒎酮(b)的合成方法 参照 a. 无色透明晶体，产率 $78.4 \%$, 纯度为 $99.0 \%$ (GC). m.p. $125.8 \sim 126.3{ }^{\circ} \mathrm{C} ;[\alpha]_{\mathrm{D}}^{20}+18.0$ (c 0.5 , $\left.\mathrm{CH}_{3} \mathrm{OH}\right)$.

4-(4'-甲氧基苯亚甲基)-2-羟基-3-蒎酮(c)的合成方 法参照 a. 浅黄色针状晶体，产率 $61.3 \%$, 纯度为 $99.8 \%$ (GC). m.p. 89.2 90.1 ${ }^{\circ} \mathrm{C} ;[\alpha]_{\mathrm{D}}^{20}-17.6\left(c 0.5, \mathrm{CH}_{3} \mathrm{OH}\right)$. 
4-(4'-羟基苯亚甲基)-2-羟基-3-蒎酮(d)的合成: 8.00 $\mathrm{g}$ 叔丁醇钾, $30 \mathrm{~mL}$ 叔丁醇, 回流反应 $11 \mathrm{~h}$, 其他条件参 照 a. 白色固体, 产率 $57.4 \%$, 纯度为 $99.7 \%$ (GC). m.p. $204.9 \sim 205.5{ }^{\circ} \mathrm{C} ;[\alpha]_{\mathrm{D}}^{20}-15.6\left(c 0.5, \mathrm{CH}_{3} \mathrm{OH}\right)$.

4-(4'-氯苯亚甲基)-2-羟基-3-蒎酮(e)的合成：以 10 $\mathrm{mL} 20 \% \mathrm{NaOH}$ 溶液代替叔丁醇钾和叔丁醇, 其他条件 参照 a. 无色透明晶体, 产率 $74.6 \%$, 纯度为 $99.7 \%$ (GC). m.p. $103.9 \sim 105.0{ }^{\circ} \mathrm{C} ; \quad[\alpha]_{\mathrm{D}}^{20}+11.6$ (c) 0.5 , $\left.\mathrm{CH}_{3} \mathrm{OH}\right)$.

4-(4'-硝基苯亚甲基)-2-羊基-3-蒎酮(f)的合成：以 $0.31 \mathrm{~g}$ (醛酮总质量的 $9 \%$ )乙醇钠, $15 \mathrm{~mL}$ 无水乙醇分别 代替叔丁醇钾和叔丁醇, 常温 $\left(20 \pm 5{ }^{\circ} \mathrm{C}\right)$ 下反应 $2 \mathrm{~h}$, 其 他条件参照 a. 浅黄色针状晶体, 产率 $83.3 \%$, 纯度为 99.1\% (GC). m.p. $164.6 \sim 166.1{ }^{\circ} \mathrm{C} ;[\alpha]_{\mathrm{D}}^{20}-57.8$ (c 0.5 , $\left.\mathrm{CH}_{3} \mathrm{OH}\right)$.

4-(呋喃-2'-亚甲基)-2-羟基-3-蒎酮 (g) 的合成：以 $0.28 \mathrm{~g}$ (醛酮总质量的 $10 \%$ )乙醇钠, $10 \mathrm{~mL}$ 无水乙醇分别 代替叔丁醇钾和叔丁醇, 常温 $\left(20 \pm 5{ }^{\circ} \mathrm{C}\right)$ 下反应 $1 \mathrm{~h}$, 其 他条件参照 a. 浅红色透明晶体, 产率 $42.3 \%$, 纯度为 $100.0 \%$ (GC). m.p. $97.3 \sim 97.5{ }^{\circ} \mathrm{C} ;[\alpha]_{\mathrm{D}}^{20}-16.6$ (c 0.5 , $\left.\mathrm{CH}_{3} \mathrm{OH}\right)$.

\section{4 化合物 $\mathrm{a} \sim \mathrm{g}$ 的紫外吸收特征及光稳定性测试}

\section{4 .1 紫外吸收范围及最大吸收波长测定}

将合成的 7 个化合物 $\mathbf{a} \sim \mathbf{g}$ 分别溶于质量分数为 $55 \%$ 乙醇水溶液中, 配成质量分数为 $0.0015 \%$ 的溶液, 在 200 600 nm 范围内进行全扫描, 测定各化合物的紫 外吸收范围, 确定最大吸收波长, 并根据下式计算出它 们的摩尔吸光系数 $\varepsilon^{[18]}$. 筛选出具有优良紫外吸收的紫 外吸收剂, 进行后续稳定性实验.

$$
\varepsilon=A / C L
$$

表 1 4-芳亚甲基-2-羊基-3-蒎酮衍生物的 ${ }^{1} \mathrm{H} \mathrm{NMR}$ 和 ${ }^{13} \mathrm{C} \mathrm{NMR}$ 分析结果

Table $1{ }^{1} \mathrm{H}$ NMR and ${ }^{13} \mathrm{C}$ NMR spectral data of the 4-arylidene-2-hydroxy-3-pinanone derivatives

\begin{tabular}{cc}
\hline Compd. & ${ }^{1} \mathrm{H}$ NMR $\left(500 \mathrm{MHz}, \mathrm{DMSO}-d_{6}\right) \delta$ \\
\hline & 蒎烷基: $0.85\left(\mathrm{~s}, 3 \mathrm{H}, 9-\mathrm{CH}_{3}\right), 1.39\left(\mathrm{~s}, 3 \mathrm{H}, 8-\mathrm{CH}_{3}\right), 1.40\left(\mathrm{~s}, 3 \mathrm{H}, 10-\mathrm{CH}_{3}\right), 1.83$ \\
& $(\mathrm{~d}, J=10.5 \mathrm{~Hz}, 1 \mathrm{H}, 7 \alpha-\mathrm{CH}), 2.11(\mathrm{t}, J=6.1 \mathrm{~Hz}, 1 \mathrm{H}, 7 \beta-\mathrm{CH}), 2.52 \sim 2.56$ \\
& $(\mathrm{~m}, 1 \mathrm{H}, 1-\mathrm{CH}), 3.28(\mathrm{t}, J=6.1 \mathrm{~Hz}, 1 \mathrm{H}, 5-\mathrm{CH}), 5.37(\mathrm{~s}, 1 \mathrm{H}, \mathrm{OH}) ;$ 苯亚甲基: \\
& $7.34 \sim 7.36\left(\mathrm{~m}, 3 \mathrm{H}, 3^{\prime}, 4^{\prime}, 5^{\prime}-\mathrm{CH}\right), 7.42\left(\mathrm{~d}, J=7.3 \mathrm{~Hz}, 2 \mathrm{H}, 2^{\prime}, 6^{\prime}-\mathrm{CH}\right), 7.50(\mathrm{~s}$, \\
& $1 \mathrm{H}, 11-\mathrm{CH})$ \\
& 蒎烷基: $0.83\left(\mathrm{~s}, 3 \mathrm{H}, 9-\mathrm{CH}_{3}\right), 1.38\left(\mathrm{~s}, 3 \mathrm{H}, 8-\mathrm{CH}_{3}\right), 1.39\left(\mathrm{~s}, 3 \mathrm{H}, 10-\mathrm{CH}_{3}\right), 1.83$ \\
& $(\mathrm{~d}, J=10.45 \mathrm{~Hz}, 1 \mathrm{H}, 7 \alpha-\mathrm{CH}), 2.10(\mathrm{t}, J=6.1 \mathrm{~Hz}, 1 \mathrm{H}, 7 \beta-\mathrm{CH}), 2.51 \sim 2.55$ \\
& $(\mathrm{~m}, 1 \mathrm{H}, 1-\mathrm{CH}), 3.29(\mathrm{t}, J=6.1 \mathrm{~Hz}, 1 \mathrm{H}, 5-\mathrm{CH}), 5.35(\mathrm{~s}, 1 \mathrm{H}, \mathrm{OH}) ;$ 苯亚甲基: \\
& $2.32(\mathrm{~s}, 3 \mathrm{H}, \mathrm{ArCH}), 7.20 \sim 7.25\left(\mathrm{~m}, 4 \mathrm{H}, 2^{\prime}, 3^{\prime}, 5^{\prime}, 66^{\prime}-\mathrm{CH}\right), 7.47(\mathrm{~s}, 1 \mathrm{H}, 11-\mathrm{CH})$ \\
& \\
& 蒎烷基: $\left.0.83(\mathrm{~s}, 3 \mathrm{H}, 9-\mathrm{CH}), 1.37(\mathrm{~s}, 3 \mathrm{H}, 8-\mathrm{CH})_{3}\right), 1.40\left(\mathrm{~s}, 3 \mathrm{H}, 10-\mathrm{CH}_{3}\right), 1.81$ \\
& $(\mathrm{~d}, J=10.4 \mathrm{~Hz}, 1 \mathrm{H}, 7 \alpha-\mathrm{CH}), 2.09(\mathrm{t}, J=6.1 \mathrm{~Hz}, 1 \mathrm{H}, 7 \beta-\mathrm{CH}), 2.52 \sim 2.54$ \\
& $(\mathrm{~m}, 1 \mathrm{H}, 1-\mathrm{CH}), 3.31(\mathrm{t}, J=6.1 \mathrm{~Hz}, 1 \mathrm{H}, 5-\mathrm{CH}), 5.31(\mathrm{~s}, 1 \mathrm{H}, \mathrm{OH}) ;$ 苯亚甲基: \\
& $3.79(\mathrm{~s}, 3 \mathrm{H}, \mathrm{ArOCH}), 6.98\left(\mathrm{~d}, J=8.75 \mathrm{~Hz}, 2 \mathrm{H}, 3^{\prime}, 5^{\prime}-\mathrm{CH}\right), 7.33(\mathrm{~d}, J=8.7$ \\
& $\left.\mathrm{Hz}, 2 \mathrm{H}, 2{ }^{\prime}, 66^{\prime}-\mathrm{CH}\right), 7.44(\mathrm{~s}, 1 \mathrm{H}, 11-\mathrm{CH})$ \\
\hline &
\end{tabular}

$A$ 为吸光度; $\varepsilon$ 为摩尔吸光系数, $\mathrm{L} \cdot \mathrm{mol}^{-1} \cdot \mathrm{cm}^{-1} ; C$ 为 样品的浓度, $\mathrm{mol} / \mathrm{L} ; L$ 为光路的长度, $\mathrm{cm}$.

\subsection{2 光稳定性测定}

各取第 1.4.1 节中配成的样品溶液 $100 \mathrm{~mL}$, 分别注 入 3 个相同规格的具塞雉形瓶中, 1 个置于阳光下, 1 个 置于室内, 1 个避光保存. 以质量分数为 $55 \%$ 的乙醇水 溶液作参比, 每天测定其在最大吸收波长处的吸光度, 比较化合物 $\mathbf{a} \sim \mathbf{g}$ 的光稳定性 ${ }^{[1,20]}$.

\section{2 结果与讨论}

\section{1 化合物 $\mathrm{a} \sim \mathrm{g}$ 结构表征} 的结构经 ${ }^{1} \mathrm{H}$ NMR, ${ }^{13} \mathrm{C}$ NMR, FT-IR 和 GC-MS 分析, 其 结果见表 1 3 所示.

\subsection{1 ${ }^{1} \mathrm{H}$ NMR 和 ${ }^{13} \mathrm{C}$ NMR 图谱}

在 ${ }^{1} \mathrm{H}$ NMR 中(表 1), 化合物 $\mathbf{a} \sim \mathbf{g}$ 蒎烷基上 $\mathrm{CH}_{3}$, $\mathrm{CH}_{2}$ 和 $\mathrm{CH}$ 的质子化学位移出现在 $\delta 0.82 \sim 3.33$ 范围内; 醇羟基的质子信号在 $\delta 5.37$ 附近出现单峰; 而苯环上 $\mathrm{CH}_{3}, \mathrm{OCH}_{3}$ 的质子信号分别在 $\delta 2.10,3.79$ 处出现比较锐 的单峰; 酚羟基的质子信号则出现在 $\delta 9.79$ 左右; $\mathrm{C}=\mathrm{C}$ 上烯氢的化学位移出现在 $\delta 7.19 \sim 7.51$ 范围内.

在 ${ }^{13} \mathrm{C}$ NMR 中(表 1), 化合物 $\mathbf{a} \sim \mathbf{g}$ 蒎烷基上饱和碳 化学位移出现在 $\delta 23.70 \sim 50.40$ 范围内; 与 $\mathrm{OH}$ 相连的 碳化学位移在 $\delta 74.67$ 附近; 羰基碳的化学位移出现在 $\delta$ 201.04 左右; 苯环上 $\mathrm{CH}_{3}, \mathrm{OCH}_{3}$ 碳核的化学位移分别在 $\delta 20.79,55.10$ 处, 而与 $\mathrm{NO}_{2}, \mathrm{OCH}_{3}, \mathrm{OH}$ 相连碳核的化学 位移出现在 $\delta 146.66,159.39,158.00$ 处, 苯环上其它碳 核的化学位移在 $\delta 114.03 \sim 132.89$ 之间. 呋喃环上碳核 的化学位移在 $\delta 112.24 \sim 151.13$ 之间.

${ }^{13} \mathrm{C}$ NMR (500 MHz, DMSO- $\left.d_{6}\right)$

$23.74,25.09,26.80,28.28,39.50$, $42.71,50.50,74.68,128.25,128.47$, $129.24,131.74,134.94,140.81$, 201.24

$20.79,23.71,25.10,26.83,28.29$, $39.50,42.73,50.53,74.68,129.06$, $129.25,131.82,132.08,137.92$, $140.16,201.20$

$23.71,25.12,26.86,28.35,39.48$, 42.66, 50.54, 55.10, 74.69, 114.03, $127.23,131.00,131.69,139.05$, $159.39,201.20$ 


\begin{tabular}{|c|c|c|}
\hline Compd. & ${ }^{1} \mathrm{H}$ NMR $\left(500 \mathrm{MHz}\right.$, DMSO- $\left.d_{6}\right) \delta$ & ${ }^{13} \mathrm{C}$ NMR $\left(500 \mathrm{MHz}\right.$, DMSO- $\left.d_{6}\right) \delta$ \\
\hline d & $\begin{array}{l}\text { 蒎烷基: } 0.83\left(\mathrm{~s}, 3 \mathrm{H}, 9-\mathrm{CH}_{3}\right), 1.37\left(\mathrm{~s}, 3 \mathrm{H}, 8-\mathrm{CH}_{3}\right), 1.40\left(\mathrm{~s}, 3 \mathrm{H}, 10-\mathrm{CH}_{3}\right), 1.80 \\
(\mathrm{~d}, J=10.4 \mathrm{~Hz}, 1 \mathrm{H}, 7 \alpha-\mathrm{CH}), 2.09(\mathrm{t}, J=6.1 \mathrm{~Hz}, 1 \mathrm{H}, 7 \beta-\mathrm{CH}), 2.50 \sim 2.55 \\
(\mathrm{~m}, 1 \mathrm{H}, 1-\mathrm{CH}), 3.33(\mathrm{t}, J=6.1 \mathrm{~Hz}, 1 \mathrm{H}, 5-\mathrm{CH}), 5.27(\mathrm{~s}, 1 \mathrm{H}, \mathrm{OH}) \text {; 苯亚甲基: } \\
6.82 \sim 6.85\left(\mathrm{~m}, 2 \mathrm{H}, 3^{\prime}, 5^{\prime}-\mathrm{CH}\right), 7.24\left(\mathrm{~d}, J=8.6 \mathrm{~Hz}, 2 \mathrm{H}, 2^{\prime}, 6^{\prime}-\mathrm{CH}\right), 7.41(\mathrm{~s}, 1 \mathrm{H} \text {, } \\
11-\mathrm{CH}), 9.79(\mathrm{~s}, 1 \mathrm{H}, \mathrm{ArOH})\end{array}$ & $\begin{array}{l}23.78,25.22,26.96,28.50,39.51, \\
42.71,50.62,74.78,115.52,125.71, \\
131.27,132.28,138.27,158.00 \\
201.29\end{array}$ \\
\hline e & $\begin{array}{l}\text { 蒎烷基: } 0.83\left(\mathrm{~s}, 3 \mathrm{H}, 9-\mathrm{CH}_{3}\right), 1.37\left(\mathrm{~s}, 3 \mathrm{H}, 8-\mathrm{CH}_{3}\right), 1.38\left(\mathrm{~s}, 3 \mathrm{H}, 10-\mathrm{CH}_{3}\right), 1.83 \\
(\mathrm{~d}, J=10.55 \mathrm{~Hz}, 1 \mathrm{H}, 7 \alpha-\mathrm{CH}), 2.10(\mathrm{t}, J=6.1 \mathrm{~Hz}, 1 \mathrm{H}, 7 \beta-\mathrm{CH}), 2.51 \sim 2.56 \\
(\mathrm{~m}, 1 \mathrm{H}, 1-\mathrm{CH}), 3.22(\mathrm{t}, J=6.1 \mathrm{~Hz}, 1 \mathrm{H}, 5-\mathrm{CH}), 5.39(\mathrm{~s}, 1 \mathrm{H}, \mathrm{OH}) \text {; 苯亚甲基: } \\
7.36\left(\mathrm{~d}, J=8.45 \mathrm{~Hz}, 2 \mathrm{H}, 3^{\prime}, 5^{\prime}-\mathrm{CH}\right), 7.44\left(\mathrm{~d}, J=8.2 \mathrm{~Hz}, 2 \mathrm{H}, 2^{\prime}, 6^{\prime}-\mathrm{CH}\right), 7.46 \\
(\mathrm{~s}, 1 \mathrm{H}, 11-\mathrm{CH})\end{array}$ & $\begin{array}{l}23.70,25.05,26.76,28.20,39.50, \\
42.70,50.46,74.65,128.48,130.41, \\
130.99,132.89,133.80,141.38, \\
201.07\end{array}$ \\
\hline f & $\begin{array}{l}\text { 蒎烷基: } 0.82\left(\mathrm{~s}, 3 \mathrm{H}, 9-\mathrm{CH}_{3}\right), 1.36\left(\mathrm{~s}, 3 \mathrm{H}, 8-\mathrm{CH}_{3}\right), 1.38\left(\mathrm{~s}, 3 \mathrm{H}, 10-\mathrm{CH}_{3}\right), 1.83 \\
(\mathrm{~d}, J=10.65 \mathrm{~Hz}, 1 \mathrm{H}, 7 \alpha-\mathrm{CH}), 2.09(\mathrm{t}, J=6.1 \mathrm{~Hz}, 1 \mathrm{H}, 7 \beta-\mathrm{CH}), 2.50 \sim 2.56 \\
(\mathrm{~m}, 1 \mathrm{H}, 1-\mathrm{CH}), 3.20(\mathrm{t}, J=6.05 \mathrm{~Hz}, 1 \mathrm{H}, 5-\mathrm{CH}), 5.44(\mathrm{~s}, 1 \mathrm{H}, \mathrm{OH}) \text {; 苯亚甲 } \\
\text { 基: } 7.51(\mathrm{~s}, 1 \mathrm{H}, 11-\mathrm{CH}), 7.58\left(\mathrm{~d}, J=8.8 \mathrm{~Hz}, 2 \mathrm{H}, 3^{\prime}, 5^{\prime}-\mathrm{CH}\right), 8.21(\mathrm{~d}, J=8.75 \\
\left.\mathrm{Hz}, 2 \mathrm{H}, 2^{\prime}, 6^{\prime}-\mathrm{CH}\right)\end{array}$ & $\begin{array}{l}23.72,25.00,26.69,28.11,39.50 \\
42.91,50.40,74.68,123.51,129.47 \\
130.40,141.94,143.54,146.66, \\
200.99\end{array}$ \\
\hline g & $\begin{array}{l}\text { 蒎烷基: } 0.79\left(\mathrm{~s}, 3 \mathrm{H}, 9-\mathrm{CH}_{3}\right), 1.37\left(\mathrm{~s}, 3 \mathrm{H}, 8-\mathrm{CH}_{3}\right), 1.42\left(\mathrm{~s}, 3 \mathrm{H}, 10-\mathrm{CH}_{3}\right), 1.82 \\
(\mathrm{~d}, J=10.55 \mathrm{~Hz}, 1 \mathrm{H}, 7 \alpha-\mathrm{CH}), 2.09(\mathrm{t}, J=6.1 \mathrm{~Hz}, 1 \mathrm{H}, 7 \beta-\mathrm{CH}), 2.53 \sim 2.58 \\
(\mathrm{~m}, 1 \mathrm{H}, 1-\mathrm{CH}), 3.72(\mathrm{t}, J=6.15 \mathrm{~Hz}, 1 \mathrm{H}, 5-\mathrm{CH}), 5.33(\mathrm{~s}, 1 \mathrm{H}, \mathrm{OH}) \text {; 呋喃亚 } \\
\text { 甲基: } 6.59\left(\mathrm{dd}, J=3.45,1.80 \mathrm{~Hz}, 1 \mathrm{H}, 4^{\prime}-\mathrm{CH}\right), 6.86(\mathrm{~d}, J=3.4 \mathrm{~Hz}, 1 \mathrm{H} \text {, } \\
\text { 5'-CH) }^{-} 7.19(\mathrm{~s}, 1 \mathrm{H}, 11-\mathrm{CH}), 7.79\left(\mathrm{~d}, J=1.65 \mathrm{~Hz}, 1 \mathrm{H}, 3^{\prime}-\mathrm{CH}\right)\end{array}$ & $\begin{array}{l}23.77,25.09,26.99,28.13,39.43, \\
43.42,50.56,74.67,112.24,116.69, \\
118.26,137.23,144.90,151.13, \\
201.04\end{array}$ \\
\hline
\end{tabular}

表 2 4-芳亚甲基-2-羊基-3-蒎酮衍生物的 IR 分析结果

Table 2 IR data of the 4-arylidene-2-hydroxy-3-pinanone derivatives

\begin{tabular}{|c|c|}
\hline Compd. & $\mathrm{IR}(\mathrm{KBr}) v / \mathrm{cm}^{-1}$ \\
\hline a & $\begin{array}{l}3474\left(v_{\mathrm{O}-\mathrm{H}}\right), 2974\left(v_{\mathrm{ar} \mathrm{C}-\mathrm{H}}, \mathrm{CH}_{3}\right), 2914\left(v_{\mathrm{as} C}-\mathrm{H}, \mathrm{CH}_{2}\right), 2866\left(v_{\mathrm{s} C}-\mathrm{H}, \mathrm{CH}_{2}\right), 1697\left(v_{\mathrm{C}=\mathrm{O}}\right), 1613\left(v_{\mathrm{C}=\mathrm{C}}\right), 1493,1469,1446 \\
\left(v_{\mathrm{C}=\mathrm{C}} \text {, 苯环 } \mathrm{C}=\mathrm{C} \text { 环伸缩振动 }\right), 1050,1011\left(\delta_{\mathrm{C}-\mathrm{H}} \text {, 苯环 } \mathrm{C}-\mathrm{H} \text { 面内弯曲振动 }\right), 838,771\left(\delta_{\mathrm{C}-\mathrm{H}} \text {, 苯环 } \mathrm{C} \text { - } \mathrm{H} \text { 面外弯 }\right. \\
\text { 曲振动 })\end{array}$ \\
\hline b & $\begin{array}{l}3474\left(v_{\mathrm{O}-\mathrm{H}}\right), 2975\left(v_{\mathrm{as} \mathrm{C}-\mathrm{H}}, \mathrm{CH}_{3}\right), 2920\left(v_{\mathrm{as} \mathrm{C}-\mathrm{H}}, \mathrm{CH}_{2}\right), 2869\left(v_{\mathrm{s} C}-\mathrm{H}, \mathrm{CH}_{2}\right), 1697\left(v_{\mathrm{C}=\mathrm{O}}\right), 1611\left(v_{\mathrm{C}=\mathrm{C}}\right), 1509,1469,1446 \\
\left(v_{\mathrm{C}=\mathrm{C}} \text {, 苯环 } \mathrm{C}=\mathrm{C} \text { 环伸缩振动 }\right), 1052,1011\left(\delta_{\mathrm{C}-\mathrm{H}} \text {, 苯环 } \mathrm{C}-\mathrm{H} \text { 面内弯曲振动 }\right), 841,811\left(\delta_{\mathrm{C}-\mathrm{H}} \text {, 苯环 } \mathrm{C} \text { - } \mathrm{H} \text { 面外弯 }\right. \\
\text { 曲振动 })\end{array}$ \\
\hline c & $\begin{array}{l}3476\left(v_{\mathrm{O}-\mathrm{H}}\right), 2974\left(v_{\mathrm{ar} \mathrm{C}-\mathrm{H}}, \mathrm{CH}_{3}\right), 2934\left(v_{\mathrm{as} \mathrm{C}-\mathrm{H}}, \mathrm{CH}_{2}\right), 2837\left(v_{\mathrm{s}-}-\mathrm{H}, \mathrm{CH}_{2}\right), 1694\left(v_{\mathrm{C}=\mathrm{O}}\right), 1602\left(v_{\mathrm{C}=\mathrm{C}}\right), 1509,1464,1443 \\
\left(v_{\mathrm{C}=\mathrm{C}} \text {, 苯环 } \mathrm{C}=\mathrm{C} \text { 环伸缩振动 }\right), 1052,1032\left(\delta_{\mathrm{C}-\mathrm{H}} \text {, 苯环 } \mathrm{C}-\mathrm{H} \text { 面内弯曲振动 }\right), 839\left(\delta_{\mathrm{C}-\mathrm{H}} \text {, 苯环 } \mathrm{C}-\mathrm{H} \text { 面外弯曲振 }\right. \\
\text { 动 })\end{array}$ \\
\hline d & $\begin{array}{l}3410\left(v_{\mathrm{O}-\mathrm{H}}\right), 2974\left(v_{\mathrm{as} \mathrm{C}-\mathrm{H}}, \mathrm{CH}_{3}\right), 2914\left(v_{\mathrm{as} \mathrm{C}-\mathrm{H}}, \mathrm{CH}_{2}\right), 2866\left(v_{\mathrm{s} C}-\mathrm{H}, \mathrm{CH}_{2}\right), 1683\left(v_{\mathrm{C}=\mathrm{O}}\right), 1594\left(v_{\mathrm{C}=\mathrm{C}}\right), 1511,1437,1371 \\
\left(v_{\mathrm{C}=\mathrm{C}} \text {, 苯环 } \mathrm{C}=\mathrm{C} \text { 环伸缩振动 }\right), 1050,1008\left(\delta_{\mathrm{C}-\mathrm{H}} \text {, 苯环 } \mathrm{C}-\mathrm{H} \text { 面内弯曲振动 }\right), 832,758\left(\delta_{\mathrm{C}-\mathrm{H}} \text {, 苯环 } \mathrm{C} \text { - } \mathrm{H} \text { 面外弯 }\right. \\
\text { 曲振动 })\end{array}$ \\
\hline e & $\begin{array}{l}3468\left(v_{\mathrm{O}-\mathrm{H}}\right), 2973\left(v_{\mathrm{as}-\mathrm{H}}, \mathrm{CH}_{3}\right), 2917\left(v_{\mathrm{as} C-\mathrm{H}}, \mathrm{CH}_{2}\right), 2866\left(v_{\mathrm{s}-}-\mathrm{H}, \mathrm{CH}_{2}\right), 1698\left(v_{\mathrm{C}=\mathrm{O}}\right), 1615\left(v_{\mathrm{C}=\mathrm{C}}\right), 1488,1402,1370 \\
\left(v_{\mathrm{C}=\mathrm{C}} \text {, 苯环 } \mathrm{C}=\mathrm{C} \text { 环伸缩振动 }\right), 1094\left(v_{\mathrm{C}-\mathrm{Cl}}\right), 1051,1012\left(\delta_{\mathrm{C}-\mathrm{H}} \text {, 苯环 } \mathrm{C}-\mathrm{H} \text { 面内弯曲振动 }\right), 840,800\left(\delta_{\mathrm{C}-\mathrm{H}} \text {, 苯环 }\right. \\
\mathrm{C} \text { - } \mathrm{H} \text { 面外弯曲振动 })\end{array}$ \\
\hline f & $\begin{array}{l}3522\left(v_{\mathrm{O}-\mathrm{H}}\right), 3116\left(v_{=\mathrm{C}-\mathrm{H}}\right), 2974\left(v_{\mathrm{as} \mathrm{C}-\mathrm{H}}, \mathrm{CH}_{3}\right), 2934\left(v_{\mathrm{as} C}-\mathrm{H}, \mathrm{CH}_{2}\right), 2912\left(v_{\mathrm{s} \mathrm{C}-\mathrm{H}}, \mathrm{CH}_{3}\right), 2866\left(v_{\mathrm{s}-}-\mathrm{H}, \mathrm{CH}_{2}\right), 1697 \\
\left(v_{\mathrm{C}=\mathrm{O}}\right), 1611\left(v_{\mathrm{C}=\mathrm{C}}\right), 1515\left(v_{\mathrm{as} \mathrm{NO}}\right), 1341\left(v_{\mathrm{s} \mathrm{NO}_{2}}\right), 1485,1392,1368\left(v_{\mathrm{C}=\mathrm{C}} \text {, 苯环 } \mathrm{C}=\mathrm{C} \text { 环伸缩振动 }\right), 1046,1008\left(\delta_{\mathrm{C}-\mathrm{H}} \text {, }\right. \\
\text { 苯环 } \mathrm{C}-\mathrm{H} \text { 面内弯曲振动 }), 850,768\left(\delta_{\mathrm{C}-\mathrm{H}} \text {, 苯环 } \mathrm{C}-\mathrm{H} \text { 面外弯曲振动 }\right)\end{array}$ \\
\hline g & $\begin{array}{l}3470\left(v_{\mathrm{O}-\mathrm{H}}\right), 3119\left(v_{\mathrm{C}-\mathrm{C}}\right), 2975\left(v_{\mathrm{as} \mathrm{C}-\mathrm{H}}, \mathrm{CH}_{3}\right), 2931\left(v_{\mathrm{as} C}-\mathrm{H}, \mathrm{CH}_{2}\right), 2919\left(v_{\mathrm{s} C}-\mathrm{H}, \mathrm{CH}_{3}\right), 2870\left(v_{\mathrm{sC}-\mathrm{H}}, \mathrm{CH}_{2}\right), 1693 \\
\left(v_{\mathrm{C}=\mathrm{O}}\right), 1607\left(v_{\mathrm{C}=\mathrm{C}}\right), 1548,1472,1371\left(v_{\mathrm{C}=\mathrm{C}} \text {, 呋喃环 } \mathrm{C}=\mathrm{C} \text { 环伸缩振动 }\right), 1053,1011\left(\delta_{\mathrm{C}-\mathrm{H}} \text {, 呋喃环上 } \mathrm{C}-\mathrm{H} \text { 面内弯 }\right. \\
\text { 曲振动 }), 745\left(\delta_{\mathrm{C}-\mathrm{H}} \text {, 呋喃环 } \mathrm{C}-\mathrm{H} \text { 面外弯曲振动 }\right)\end{array}$ \\
\hline
\end{tabular}


表 3 4-芳亚甲基-2-羟基-3-蒎酮衍生物的 MS 分析结果

Table 3 MS data of the 4-arylidene-2-hydroxy-3-pinanone derivatives

\begin{tabular}{|c|c|}
\hline Compd. & MS $(70 \mathrm{eV}) m / z(\%)$ \\
\hline a & $\begin{array}{l}256\left(\mathrm{M}^{+}, 10\right), 238\left(\mathrm{M}^{+}-\mathrm{H}_{2} \mathrm{O}, 7\right), 223\left(\mathrm{M}^{+}-\mathrm{H}_{2} \mathrm{O}-\mathrm{CH}_{3}, 3\right), 213\left(\mathrm{M}^{+}-\mathrm{C}_{3} \mathrm{H}_{7}, 29\right), 187(13), 157(26), 129(40), 115 \\
(27), 99(100), 91(37), 77(14), 65(6), 55(7)\end{array}$ \\
\hline b & $\begin{array}{l}270\left(\mathrm{M}^{+}, 11\right), 252\left(\mathrm{M}^{+}-\mathrm{H}_{2} \mathrm{O}, 6\right), 237(4), 227(29), 212(9), 201(21), 199(6), 185(5), 171(38), 165(8), 157(14), \\
143(31), 141(20), 129(25), 115(23), 105(32), 99 \text { (100), } 91 \text { (12), } 79(12), 65(6)\end{array}$ \\
\hline c & $\begin{array}{l}286\left(\mathrm{M}^{+}, 24\right), 268(5), 244(7), 243(37), 228 \text { (13), } 217(87), 187 \text { (100), } 173 \text { (10), } 159 \text { (44), } 145(20), 121(47), 115 \\
(33), 99(72), 91(13), 79(13), 77(15), 55(8)\end{array}$ \\
\hline d & $\begin{array}{l}272\left(\mathrm{M}^{+}, 18\right), 254(6), 229(38), 214(14), 203(54), 187(7), 173 \text { (64), } 159 \text { (11), } 145 \text { (43), } 131(30), 127(31), 115 \\
(44), 107(51), 99(100), 91(21), 79(19), 77 \text { (30), } 55 \text { (14) }\end{array}$ \\
\hline e & $\begin{array}{l}290\left(\mathrm{M}^{+}, 7\right), 272(4), 247(21), 234 \text { (3), } 221 \text { (13), } 191 \text { (17), } 165 \text { (11), } 141 \text { (10), } 127 \text { (20), } 99 \text { (100), } 81 \text { (7), } 77 \text { (6), } 55 \\
(6)\end{array}$ \\
\hline f & $301\left(\mathrm{M}^{+}, 14\right), 283(19), 258(33), 202(36), 156(20), 141(38), 99$ (100), 91 (13), $81(17), 77(23), 71$ (27), 55 (20) \\
\hline g & $\begin{array}{l}246\left(\mathrm{M}^{+}, 23\right), 228(10), 213(3), 203(46), 188(12), 177 \text { (46), } 159 \text { (11), } 147 \text { (44), } 133 \text { (8), } 119 \text { (16), } 99 \text { (100), } 81 \text { (33), } \\
77(16), 65(18), 55(15)\end{array}$ \\
\hline
\end{tabular}

\subsubsection{IR 图谱}

化合物 $\mathbf{a} \sim \mathbf{g}$ 的红外光谱在 $3410 \sim 3522 \mathrm{~cm}^{-1}$ 附近均 出现 $\mathrm{OH}$ 的伸缩振动吸收峰; 在 $2866 \sim 2980 \mathrm{~cm}^{-1}$ 之间 为甲基及亚甲基碳氢键伸缩振动吸收峰; 在 1683 $1698 \mathrm{~cm}^{-1}$ 范围内有 $\mathrm{C}=\mathrm{O}$ 伸缩振动吸收峰; 在 1594 $1615 \mathrm{~cm}^{-1}$ 有较强的 $\mathrm{C}=\mathrm{C}$ 的伸缩振动吸收峰; 在 $1464 \sim 1591 \mathrm{~cm}^{-1}$ 区域均有苯环的骨架的伸缩振动吸收 峰; 在 1046 1053 $\mathrm{cm}^{-1}$ 之间有 $\mathrm{C}-\mathrm{O}$ 键的伸缩振动吸 收峰; $771 \mathrm{~cm}^{-1}$ 左右为单取代苯环的面外弯曲或扭曲的 特征吸收; 797 839 $\mathrm{cm}^{-1}$ 区域的吸收峰为对位二取代 苯环上两个相邻的 $\mathrm{H}$ 的面外弯曲振动.

\subsubsection{MS 图谱}

EI-MS 数据, 均可见到化合物 $\mathbf{a} \sim \mathbf{g}$ 的分子离子峰, 且吻合得很好. 表 1 3 结果表明: 这些波谱数据表征了 目标化合物 $\mathbf{a} \sim \mathbf{g}$ 的结构, 与预先设计的结构完全吻合.

\section{2 化合物 $a \sim g$ 的紫外吸收特征}

由表 4 可知, 当助色基团(如 $\mathrm{OH}, \mathrm{OCH}_{3}, \mathrm{Cl}$ )或生色 基团(如 $\mathrm{NO}_{2}$ )与苯环直接相连时, 发生红移, 同时吸收 强度有所增强; 化合物 $\mathbf{a} \sim \mathbf{g}$ 的摩尔吸光系数均大于 $10^{4}$ $\mathrm{L} \cdot \mathrm{mol}^{-1} \cdot \mathrm{cm}^{-1}$, 具有较强的紫外吸收. 化合物 $\mathbf{a}, \mathbf{b}, \mathbf{e}$ 的 紫外吸收范围和最大吸收波长很接近, 但摩尔吸光系数 却有一定差别, 都可作为 UVB $(280 \sim 320 \mathrm{~nm})$ 型紫外线 吸收剂. 化合物 $\mathbf{c}$ 和 $\mathbf{d}$ 的紫外吸收范围分别为 262.0 406.0 和 $262.5 \sim 402.0 \mathrm{~nm}$, 紫外吸收特征非常相似, 紫 外线吸收范围、最大吸收波长、摩尔吸光系数都很接近, 具有同时吸收 UVA $(320 \sim 400 \mathrm{~nm})$ 和 UVB $(280 \sim 320$ $\mathrm{nm}$ )的功能. 化合物 $\mathbf{f}$ 和 $\mathbf{g}$ 的紫外吸收范围分别为 $243.0 \sim 398.0$ 和 $267.5 \sim 403.0 \mathrm{~nm}$, 最大吸收波长 $\lambda_{\max }$ 和
摩尔吸光系数分别为 $316.5 \mathrm{~nm}, 20301.91 \mathrm{~L} \cdot \mathrm{mol}^{-1} \cdot \mathrm{cm}^{-1}$ 和 $338.5 \mathrm{~nm}, 23210.72 \mathrm{~L} \cdot \mathrm{mol}^{-1} \cdot \mathrm{cm}^{-1}$, 具有同时吸收 UVA 和 UVB 的功能. 从摩尔吸光系数来看, 目标化合 物对紫外线的吸收能力顺序为 $\mathbf{g}>\mathbf{e}>\mathbf{f}>\mathbf{b}>\mathbf{d}>\mathbf{c}>\mathbf{a}$.

表 4 4-芳亚甲基-2-羟基-3-蒎酮衍生物的紫外吸收范围及最 大吸收波长

Table 4 Ranges of ultraviolet absorption and the maximum absorption wavelengths of the 4-arylidene-2-hydroxy-3-pinanone derivatives

\begin{tabular}{|c|c|c|c|}
\hline Compd. & Ultraviolet absorption $/ \mathrm{nm}$ & $\lambda_{\max } / \mathrm{nm}$ & 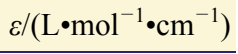 \\
\hline $\mathbf{a}$ & $243.5 \sim 361.0$ & 299.5 & 16154.32 \\
\hline b & $252.5 \sim 379.0$ & 311.0 & 19652.02 \\
\hline c & $262.0 \sim 406.0$ & 325.5 & 18701.72 \\
\hline d & $262.5 \sim 402.0$ & 328.0 & 19051.63 \\
\hline e & $247.5 \sim 367.0$ & 302.0 & 21093.24 \\
\hline f & $243.0 \sim 398.0$ & 316.5 & 20301.91 \\
\hline g & $267.5 \sim 403.0$ & 338.5 & 23210.72 \\
\hline
\end{tabular}

\section{3 化合物 $a \sim g$ 的光稳定性}

所合成的 4-芳亚甲基-2-羑基-3-蒎酮衍生物 $\mathbf{a} \sim \mathbf{g}$ 在 太阳光下的光稳定性实验结果如图 1 所示.

由图 1 可明显看出，所合成的 7 个化合物在光照下 吸光度减小, 同时各摩尔吸光系数也随之发生变化.

对化合物 $\mathbf{a} \sim \mathbf{f}$ 而言, 其摩尔吸光系数在被阳光照 射第 $1 \mathrm{~d}$ 的变化比较明显, 被阳光照射第 $1 \mathrm{~d}$ 与未被阳光 照射相比, 摩尔吸光系数分别下降了 $12.76 \%, 11.27 \%$, $5.65 \%, 0.42 \%, 9.16 \%, 9.20 \%$; 被阳光照射第 $2 \mathrm{~d}$ 与第 $1 \mathrm{~d}$ 相比, 摩尔吸光系数分别下降了 $2.01 \%, 0.46 \%, 0.96 \%$, $0.32 \%, 0.78 \%, 0.12 \%$; 而在被阳光照射第 $2 \mathrm{~d}$ 以后，它们 的摩尔吸光系数基本不发生变化, 均在 $1.22 \%$ 以内. 对 


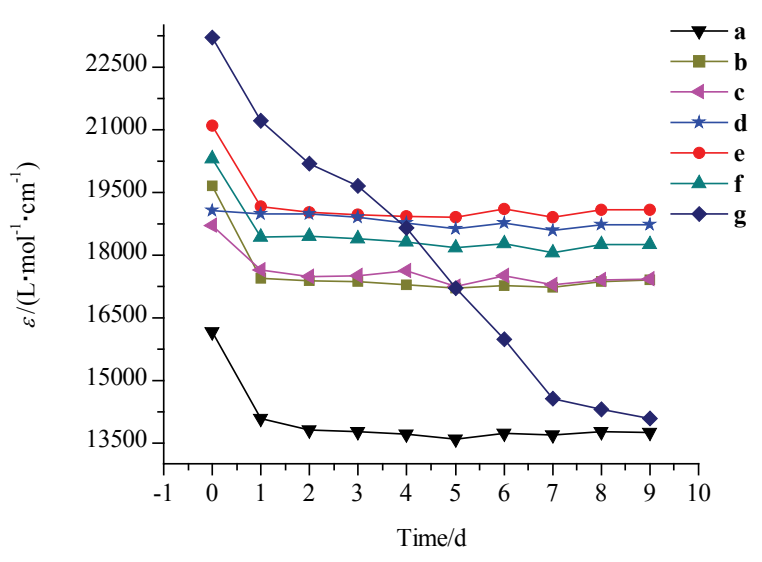

图 1 4-芳亚甲基-2-羊基-3-蒎酮衍生物在光照环境下的时间摩尔吸光系数曲线

Figure 1 Photodegradation of the 4-arylidene-2-hydroxy-3pinanone derivatives under sunlight

化合物 $\mathbf{g}$ 而言, 在光照的条件下, 摩尔吸光系数的变化 更为明显, 而且在光照环境的 $9 \mathrm{~d}$ 之内摩尔吸光系数一 直在发生变化, 分别下降了 $8.61 \%, 4.80 \%, 2.70 \%$, $5.09 \%, 7.80 \%, 7.08 \%, 8.87 \%, 1.75 \%, 1.52 \%$, 只是在光照 第 $7 \mathrm{~d}$ 之后才趋于稳定. 由此表明: 化合物的稳定性顺 序为 $\mathbf{d}>\mathbf{c}>\mathbf{e}>\mathbf{f}>\mathbf{b}>\mathbf{a}>\mathbf{g}$, 因此, 除化合物 $\mathbf{g}$, 其它 6 个化合物均具有较好的光稳定性.

以同样的实验方法, 对化合物 $\mathbf{a} \sim \mathbf{g}$ 在室内和避光 条件下的摩尔吸光系数变化情况也进行了测定, 其摩尔 吸光系数变化趋势如图 2 和图 3 所示.

由图 2 可以看出, 在室内条件下, 各化合物的吸光 度下降得并不大, 尤其是化合物 $\mathbf{g}$ 的稳定性比光照条件 下稳定得多. 在室内放置 $1 \mathrm{~d}$ 后, 化合物 $\mathbf{a} \sim \mathbf{g}$ 的摩尔吸 光系数分别下降了 $1.64 \%, 1.42 \%, 1.36 \%, 0.32 \%, 1.32 \%$, $3.61 \%, 1.02 \%$. 而在室内放置 $2 \mathrm{~d}$ 后, 它们的摩尔吸光系 数基本不发生变化, 均在 $0.31 \%$ 以内.

由图 3 可见, 在避光条件下化合物 $\mathbf{a} \sim \mathbf{g}$ 的稳定性比 光照和室内条件下更稳定. 除化合物 $\mathbf{a}$ 的摩尔吸光系数 略有下降外, 其它化合物的摩尔吸光系数反而有所提 高, 在 $0.41 \% \sim 3.08 \%$ 范围不等.

\section{3 结论}

以(一)- $\alpha$-蒎烯为原料合成了 7 个具有光学活性的新 型 4-芳亚甲基-2-羟基-3-蒎酮衍生物 $\mathbf{a} \sim \mathbf{g}$. 采用 ${ }^{1} \mathrm{H}$ $\mathrm{NMR},{ }^{13} \mathrm{C}$ NMR, GC-MS 和 FT-IR 等分析手段对所得化 合物的结构进行了鉴定, 并考察了它们的紫外吸收特性 及光稳定性. 结果表明: 化合物 $\mathbf{g}$ 的紫外吸收能力最强, 其次是 $\mathbf{e}$, 而 $\mathbf{a}$ 的紫外吸收能力相对最弱, 紫外吸收能 力顺序为 $\mathbf{g}>\mathbf{e}>\mathbf{f}>\mathbf{b}>\mathbf{d}>\mathbf{c}>\mathbf{a}$; 化合物 $\mathbf{d}$ 的光稳定性 最好, 而 $\mathbf{g}$ 的光稳定性最差, 光稳定性顺序为 $\mathbf{d}>\mathbf{c}>$

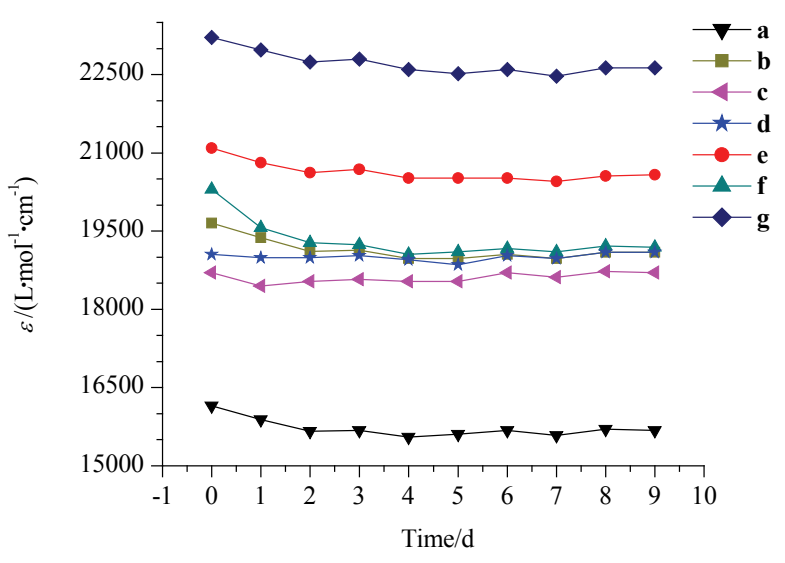

图 2 4-芳亚甲基-2-羟基-3-蒎酮衍生物在室内环境下的时间摩尔吸光系数曲线

Figure 2 Photodegradation of the 4-arylidene-2-hydroxy-3pinanone derivatives under indoor circumstance

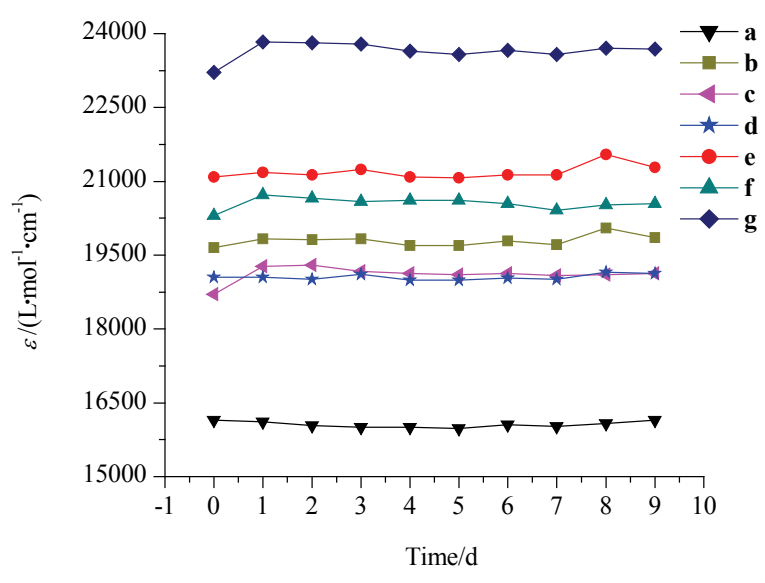

图 3 4-芳亚甲基-2-羊基-3-蒎酮衍生物在避光环境下的时间摩尔吸光系数曲线

Figure 3 Photodegradation of 4-arylidene-2-hydroxy-3pinanone derivatives in closed environment

$\mathbf{e}>\mathbf{f}>\mathbf{b}>\mathbf{a}>\mathbf{g}$. 研究结果为开发新型紫外线吸收剂提 供了一个全新的合成方法和新的原料来源.

\section{References}

[1] Wang, Y.-M.; Tang, B.-T.; Ma, W.; Zhang, S.-F. Chin. J. Org. Chem. 2010, 30, 330 (in Chinese).

(王云明, 唐炳涛, 马威, 张淑芬, 有机化学, 2010, 30, 330.)

[2] Pathak, M. A. Skin Pharmacol. 1991, 4, 85.

[3] Michihiro, H.; Kimihiko, H.; Genji, I. JP 6114053, 1987 [Chem. Abstr. 1987, 104, 172755g].

[4] Cao, Z.; Zhang, Z.-J. China Surf. Deterg. Cosmet. 2009, 39, 199 (in Chinese).

(曹智, 张治军, 日用化学工业, 2009, 39, 199.)

[5] Yu, H.-J.; Zheng, Y.-B.; Du, J.; Liu, J.-P. China Surf. Deterg. Cosmet. 2005, 35, 248 (in Chinese).

(于淑娟, 郑玉斌, 杜杰, 刘建平, 日用化学工业, 2005, 35, 248.)

[6] Ziegler, A.; Jonason, A. S.; Leffell, D. J.; Simon, J. A.; Sharma, H. 
W.; Kimmelman, J.; Remington, L.; Jacks, T.; Brash, D. E. Nature 1994, 372, 773 .

[7] Hochberg, M.; Enk, C. D. Photochem. Photobiol. 1999, 70, 766.

[8] Broadbent, J. K.; Martincigh, B. S.; Raynor, M. W.; Salter, L .F.; Moulder, R.; Sjöberg, P.; Markides, K. E. J. Chromatogr. A 1996, $732,101$.

[9] Stokes, R.; Diffey, B. Int. J. Cosmet. Sci. 1999, 21, 341.

[10] Yao, X.-Y. Chin. J. Health Lab. Technol. 2005, 15, 236 (in Chinese).

(姚孝元，中国卫生检验杂志, 2005, 15, 236.)

[11] Lang, G.; Deflandre, A.; Beck, B. DE 3402645, 1984 [Chem. Abstr. 1984, 101, 216224].

[12] Forestier, S.; Lang, G.; Sainte-Beuve, E. DE 3629241, 1989 [Chem. Abstr. 1988, 108, 43839].

[13] Lang, G.; Foxestier, S.; Lagrange, A.; Moire, C.; Deflandre, A. DE 3833706,1989 [Chem. Abstr. 1989, 111, 153349].

[14] Zhen, J. Sci. Chem. Commodity 2010, 33, 18 (in Chinese).
(郑 洁, 日用化学品科学, 2010, 33, 18.)

[15] Qian, M.-X. Chemistry 1985, (12), 28 (in Chinese). (钱铭熙, 化学通报, 1985, (12), 28.)

[16] Wang, S.-F.; Li, Y.-P.; Zhang, M.-G. Chin. J. Org. Chem. 2007, 27, 1612 (in Chinese).

(王石发, 李艳苹, 张明光, 有机化学, 2007, 27, 1612.)

[17] Yang, Y.-Q.; Li, Y.-P.; Wang, S.-F.; Gu, W. Chin. J. Org. Chem. 2009, 29, 1082 (in Chinese).

(杨益琴, 李艳苹, 王石发, 谷文, 有机化学, 2009, 29, 1082.)

[18] Liu, H.-M. Practical Spectral Analysis for Organic Compounds, Zhengzhou University Press, Zhengzhou, 2008 (in Chinese). (刘宏民, 实用有机光谱解析, 郑州大学出版社, 郑州, 2008.)

[19] Cao, J.-.F.; Zhou, J. China Surf. Deterg. Cosmet. 2004, 27, 259 (in Chinese).

(曹锦芳, 周洁, 日用化学工业, 2004, 27, 259.)

[20] Scalia, S.; Casolari, A.; Iaconinoto, A.; Simeoni, A. J. Pharm. Biomed. 2002, 30, 1181 .

(Qin, X.) 